observational studies ( 3 cross-sectional, 8 case-control and 8 cohort) and 12 were randomised controlled trials. Sixteen of the 19 observational studies reviewed reported statistically significant associations between vitamin $\mathrm{D}$ deficiency and susceptibility to ARI, and 3 reported no such association. Six of the 12 clinical trials reviewed reported protective effects of vitamin $\mathrm{D}$ against ARI, while five reported null effects, and one reported an adverse effect on pneumonia recurrence.

Conclusions Observational studies report consistent associations between vitamin $\mathrm{D}$ deficiency and susceptibility to ARI in a wide range of age-groups in diverse clinical settings. By contrast, randomised controlled trials of vitamin $\mathrm{D}$ supplementation for the prevention of ARI report conflicting results, possibly reflecting varying prevalence of vitamin $\mathrm{D}$ deficiency in study populations and/or heterogeneity in vitamin $\mathrm{D}$ supplementation regimens investigated.

\section{P20 FATIGUE AND POOR LUNG FUNCTION ARE SIGNIFICANTLY ASSOCIATED WITH IMPAIRED HEALTH-RELATED QUALITY OF LIFE (HROOL) IN A LARGE COHORT OF PATIENTS WITH CHRONIC PULMONARY ASPERGILLOSIS}

doi:10.1136/thoraxjnl-2012-202678.161

KA Al-shair, GT Atherton, DK Kennedy, GP Powell, DWD Denning. The National Aspergillosis Centre, University Hospital of South Manchester, Manchester University, Manchester, UK

Introduction Fatigue is a prominent disabling symptom in several chronic pulmonary diseases; however, its impact on HRQoL in patients with chronic pulmonary aspergillosis (CPA) has not been investigated.

Method Our 154 patients with CPA completed the Manchester COPD Fatigue Scale (MCFS), Thorax 2009)) and the SGRQ in our specialist referral centre. MCFS measures total fatigue and sub-components comprehensively. Lung function and body mass index were measured. Univariate and multivariate linear and binary analysis, and the principal component analysis (PCA) were used.

Results The mean (SD) age $(61.1$ (10.8)) years and 44\% were female; $\mathrm{FEV}_{1} \%$ (63.3 (24.9)), BMI (23.7 (5.2)), SGRQ total score (55.6 (23.5) and MCFS total score (30 (14.9)).

PCA showed that 27 items of MCFS loaded clearly on three components: physical and psychosocial and cognitive fatigue.

Univariate analysis showed a strong association between total SGRQ score and MCFS score $(r=0.81, p<0.001)$. Using total SGRO as a dependent variable, linear multi-variate analysis showed that fatigue was the strongest factor (beta $=0.83 p<0.0001$ ) associated with impaired health status followed by $\mathrm{FEV}_{1} \%$ (beta $=-0.22$, $p=0.009)$, but no statistically significant association with age, BMI and pack/years. This model explained $70 \%$ of the variance of SGRO total score.

Using patients' self-assessment grades of SGRQ (Very poor, poor, fair, good and very good), one-way ANOVA showed that patients with "very poor" health status had the highest fatigue scores (45 (6.4)), following by poor (35 (10.1)), fair (30 (10.4)), good (14 (10.9)) and very good $(0)(p<0.001)$. Splitting the group to (very poor and poor) versus (fair, good and very good), the ROC curve analysis indicated significant ability of MCFS and its components to detect change in HS (AUC $=0.82$; range $0.75-0.9, \mathrm{p}<0.0001$ ) as demonstrated in figure 1.

Furthermore, binary regression analysis showed that only fatigue score $(\mathrm{OR}=0.92,95 \% \mathrm{CI} 0.87-0.97 ; \mathrm{p}=0.002)$ and $\mathrm{FEV}_{1} \%(\mathrm{OR}=1.04$, $95 \%$ CI 1.01-1.07, $\mathrm{p}=0.02)$ are significantly associated with impaired health status after correcting to age, gender and DLCO\%. Conclusion This is the first study directly implicating fatigue as a major factor affecting health-related-quality of life in patients with CPA.
Figure 1, ROC curve analysis of the MCFS and its components; discrimination between health status categories

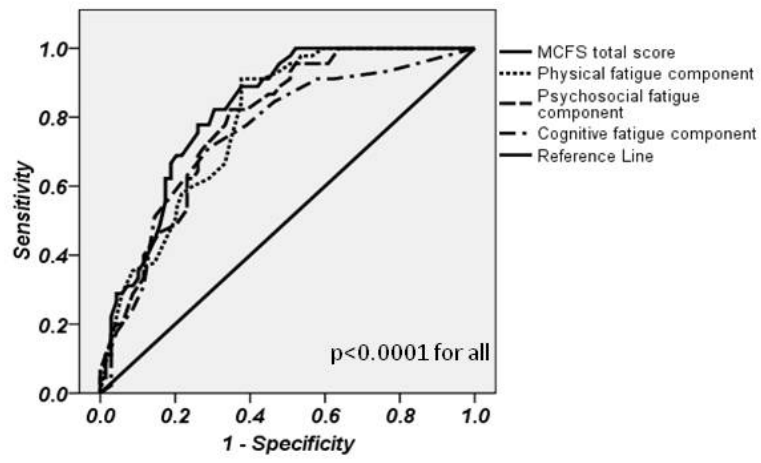

Abstract P20 Figure 1

\section{P21 LONG TERM ANTIFUNGAL TREATMENT (LTAFT) IS EFFECTIVELY ASSOCIATED WITH IMPROVEMENT IN HEALTH STATUS (HS) IN PATIENTS WITH CHRONIC PULMONARY ASPERGILLOSIS (CPA)}

doi:10.1136/thoraxjnl-2012-202678.162

KA Al-shair, MK Kirwan, GA Atherton, AC Caress, DWD Denning. The National Aspergillosis Centre, University Hospital of South Manchester, Manchester University, Manchester, UK

Introduction $\mathrm{CPA}$ is a chronic progressive respiratory infectious disease results in significant lung tissue destruction with a $50 \%+5$ year mortality. Response to antifungal therapy is slow, with $\sim 80 \%$ of patients who respond doing so by 6 months. We recently demonstrated the reliability and validity of SGRQ in examining HS in CPA (Chest, in press), and now present longitudinal data on the efficacy of LTAFT in improving HS in CPA patients.

Method HS of 98 CPA patients were assessed 3 times over 6 months using the well-established standardised SGRO. CPA severity was assessed using our published CPA banding system. $\mathrm{FEV}_{1}$, BMI, dyspnoea (using MRC dyspnoea scale) were measured.

Results Mean age was 58 years and 48\% were female; and 25, 58 and 15 had band 1, 2 and 3 CPA respectively. At visit 2 and 3 (V2 and V3), we found that overall total and domain SGRO scores were either lower (improved health status) or similar compared to V1 (table 1).

Categorizing the cohort by those who reported improvement or deterioration by a total SGRO score of $\geq 4$ at V3 comparing to V1, we found that $43 \%$ improved, $22 \%$ remained stable and $35 \%$ deteriorated. The median (IOR) of total SGRQ score of the improved group at V3 was 58 (42-66) compared to 71 (60-79) at V1; and for the deteriorated group was $67.5(57-76)$ at V3 compared to 62 $(41-67))$ at $\mathrm{V} 1$. The deteriorated were older (62 (9.8) years versus 56.1 (9.3) ( $p=0.008)$; and tended to have lower BMI, more dyspnoea and worse lung function. Moreover, binary regression multivariate analysis showed that age maintained its association with deterioration in HS (OR 1.13, 95\% CI 1.01-1.26, $\mathrm{p}=0.03$ ) after correcting for gender, $\mathrm{BMI}$ and $\mathrm{FEV}_{1} \%$.

Of the 37 patients started on an antifungal agent at V1 who took it for $3+$ months (including a 3 week IV course of amphotericin B), $22(59 \%)$ improved, 11 (30\%) were stable and 4 (11\%) deteriorated at V3.

Conclusion LTAFT prevented/reduced the progression of CPA and patients preserved overall good HS. More therapeutic approaches for this progressive disease are urgently needed. 